\title{
Single/Dual-Polarized Infrared Rectenna for Solar Energy Harvesting
}

\author{
Saber H. Zainud-Deen ${ }^{1}$, Nermeen A. Eltresy ${ }^{1}$, Hend A. Malhat ${ }^{1 *}$, and Kamal H. Awadalla ${ }^{1}$ \\ Department of Electrical Engineering \\ Faculty of Electronic Eng., Menoufia University, 32952, Egypt \\ *corresponding author, E-mail: er_honidal@yahoo.com
}

\begin{abstract}
Single and dual linearly-polarized receiving mode nanoantennas are designed for solar energy harvesting at $28.3 \mathrm{THz}$. The infrared rectennas are used to harvest the solar energy and converting it to electrical energy. The proposed infrared rectenna is a thin dipole made of gold and printed on a silicon dioxide substrate. Different shapes of the dipole arms have been investigated for maximum collected energy. The two poles of the dipole have been determined in a rectangular, circular and rhombus shapes. The rectenna dipole is used to concentrate the electromagnetic energy into a small localized area at the inner tips of the gap between the dipole arms. The dimensions of the different dipole shapes are optimized for maximum near electric field intensity at a frequency of 28.3 THz. A Metal Insulator Metal (MIM) diode is incorporated with the nanoantenna dipole to rectify the received energy. The receiving efficiency of the solar energy collector with integrated MIM diode has been investigated. A dualpolarized, four arms, rhombus shaped nanoantenna dipole for solar energy harvesting has been designed and optimized for $28.3 \mathrm{THz}$ applications.
\end{abstract}

\section{Introduction}

Lately, due to the pollution from the traditional fuel energy sources, solar energy is widely utilized as a clean energy source. The solar energy is the cleanest, greenest and environment friendly energy source. It can be utilized for the production of electric energy as there is no harmful gas emission produced [1]. Approximately $80 \%$ of the solar energy included in the $0.2-2 \mu \mathrm{m}$ spectral range is absorbed by the Earth's surface and the atmosphere. It is re-emitted as infrared radiation in the $7-17 \mu \mathrm{m}$ spectral range with peaks at 28.3 THz [2]. Artificial thermal sources emit thermal energy in the same IR band such as engines, furnaces, etc. Photovoltaic (PV) solar cell is usually used to convert thermal energy to electrical energy. But, it has many disadvantages, 1 . It has a low conversion efficiency $(20 \%)$ because it depends on the input light, the surface area, and sensitivity to the weather conditions. 2. It involves a mechanical sun-tracking system to optimize conversion.

Nanoantenna is a device designed to convert freepropagating optical radiation to a localized energy, and vice versa [3]. Nanoantennas have wide range of applications such as optical beam manipulation, energy harvesting, sensing, nonlinear optics and medical therapies [4-6]. The nanoantennas have the ability to enhance the interaction of light or infrared with a highly localized field in the gap area [7]. At the terahertz frequency range, the electrical properties of metals are described by the Drude model which considers both the free electron contributions and harmonic oscillator surface plasmon polariton resonances (SPPRs) contributions [8]. Solar energy harvesting is using nanoantennas, which absorb the propagating optical and IR radiation by an induced current in their resonant elements. The emitted energy from Earth at $7-17 \mu \mathrm{m}$ wavelength is collected by the nanoantenna and is converted to electrical energy. The solar energy nanoantenna collector has theoretically $100 \%$ energy conversion efficiency. The advantages of nanoantenna collectors are; they can work whole the day, and not sensitive to the weather conditions [9]. The frequency response of the solar energy harvesting nanoantenna depends primarily on the design of the antenna and its impedance matching to the rectifier diode [9]. The rectifiers are used to rectify currents at infrared wavelengths (without an external potential bias) are the femtosecond-fast metal/dielectric/metal (MIM) diodes, low-capacitance and low-resistance geometric diodes. Different forms of the nanoantennas such as dipole, bow-tie and spiral antenna have been investigated for solar energy collection [1-4, 9$11]$.

In this paper, different shapes of IR- rectenna dipole have been designed and optimized for maximum field intensity across the dipole gap. The variations of the electric field intensity with dipole geometric parameters have been investigated. The effect of incident plane wave polarization on the performance of IR nanoantenna is examined. All the dimensions of the antenna are optimized using CST and HFSS software packages in order to make the best performance over the frequency range of involvement. A dual-polarized, four rhombus shaped arms nanoantenna quad pole for solar energy harvesting has been designed and optimized at $28.3 \mathrm{THz}$. The finite integral technique (FIT) is used to analyze the nanoantenna solar energy collectors [12], and the finite element method (FEM) is used for confirmation of the results. 


\section{Material properties at terahertz range}

The electromagnetic radiation re-emitted by the Earth is estimated using the Stefan Boltzmann radiation law. The thermal radiation emitted by the Earth is approximated as a blackbody that absorbs all the incoming radiation and emits a temperature-dependent radiation spectrum [1]. The maximum blackbody spectral irradiance occurs close to a wavelength of $10.6 \mu \mathrm{m}$. The Earth radiates a circularly polarized plane wave and the considered antenna is linearly polarized. Thus, the received power drops to a half of the incident power. By considering the plane wave propagating power, the relation between the electric field amplitude $\mathrm{E}$ and the Earth emitted power density $p_{E}$ is $E=\sqrt{2 Z_{o} p_{E}}$ $=239 \mathrm{~V} / \mathrm{m}$, where $\mathrm{Z} 0=377 \mathrm{ohm}$ is the impedance of the free space, $p_{E}=152.5 \mathrm{~W} / \mathrm{m} 2$ considering the earth temperature $\mathrm{T}=287 \mathrm{~K}$ and the emissivity $=0.9$ [1]. The frequency dependent complex permittivity and the electrical conductivity of metals in the terahertz frequency range can be depicted by the Drude model as [3]

$$
\varepsilon_{r}=\varepsilon_{1}+j \varepsilon_{2}=1-\frac{\omega_{p}^{2}}{\omega\left(\omega-j \vartheta_{p}\right)}
$$

where $\varepsilon_{1}$ is the real part of the permittivity and is a measure of how much energy from an external field is stored in a material. $\varepsilon_{2}$ is the imaginary part of permittivity (loss factor). It is a measure of how dissipative or lossy a material is to an external field, $\varepsilon_{o}$ is the dielectric constant of vacuum, $\omega$ is the angular frequency of the electromagnetic wave, $\vartheta_{p}$ is the angular collision frequency, and $\omega_{p}$ is the electron plasma angular frequency [13]

$$
\omega_{p}=\sqrt{n_{e} q^{2} / \varepsilon_{o} m_{e}}=56.40 \sqrt{n_{e}}
$$

where $n_{e}$ is the free electron density in electrons per cubic meter, $m_{e}$ is the electron mass, and $q$ is the charge of the electron. In terahertz band the complex conductivity $\sigma$ of metals is frequency dependent and is related to the electrical dispersion by the following relation

$$
\sigma_{1}=\varepsilon_{o} \varepsilon_{2} \omega, \text { and } \sigma_{2}=\varepsilon_{o}\left(\varepsilon_{1}-1\right) \omega
$$

where $\sigma_{1}$ and $\sigma_{2}$ are the real and imaginary parts of conductivity. Figure 1a shows the variations of the relative permittivity $\varepsilon_{r}$ versus frequency for gold, copper, and silver. By increasing the frequency $\varepsilon_{1}$ is increased while $\varepsilon_{2}$ decreases in magnitude with increasing frequency. The same behaviour is observed for the electrical conductivity as shown in Fig. 1b.

\section{Numerical results}

The IR nanoantenna collector has been designed and optimized in this work for solar energy harvesting at 28.3 THz. The first IR nanoantenna consists of rhombus shaped printed dipole with side length $\mathrm{L}_{\mathrm{s}}=5 \mu \mathrm{m}$ and tip angle $\Psi=90$ degrees. The dipole is printed on $\mathrm{Sio}_{2}$ dielectric substrate with $\varepsilon_{r}=3.9$ and dimensions of $9 \times 5 \times 0.15 \mu \mathrm{m}^{3}$. The dipole is designed to receive IR radiation at a wavelength of 10.6 $\mu \mathrm{m}$. A linearly polarized incident wave launched on the upper face of the substrate and propagates along the negative z-direction with an electric field vector parallel to the $\mathrm{x}$-axis, is used to excite the nanoantenna as shown in Fig. 2. A parametric study on the nanoantenna dimensions has been carried out for maximum electric field at the dipole gap. When the antenna is excited into a resonance mode, it induces a cyclic plasmon's movement of free electrons in the nanoantenna. The electrons flow along the antenna, generating alternating current at the same frequency of the incident wave.

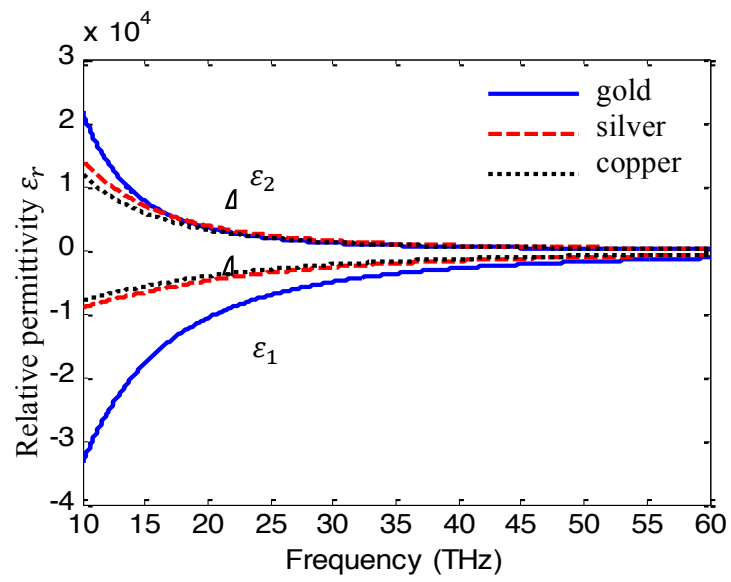

a. Electrical complex permittivity

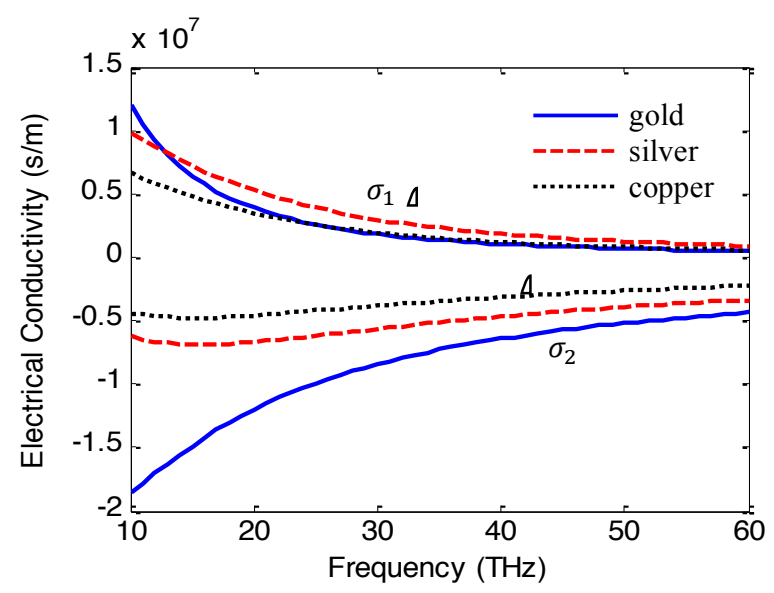

b. Electrical conductivity

Figure 1: The variations of complex permittivity and electrical conductivity versus frequency for different types of metals.

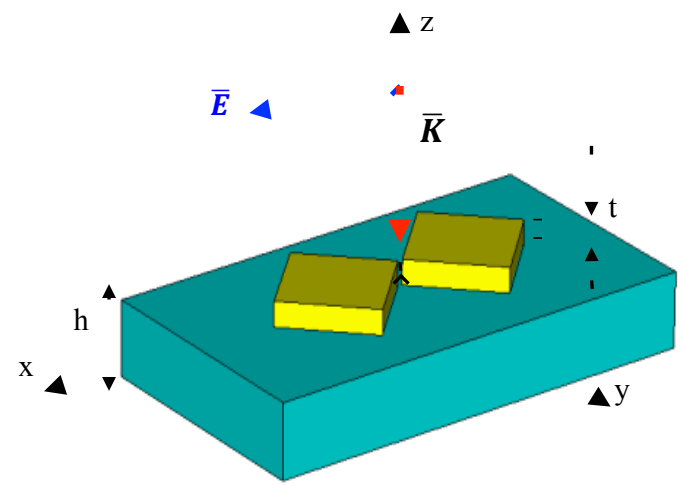

a. 3-D view 


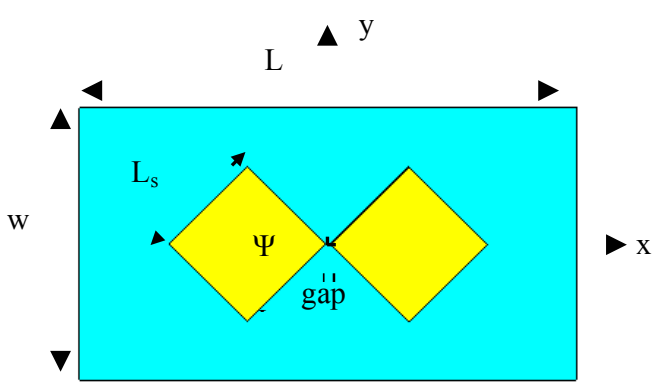

b. Top- view

Figure 2. The detailed construction of rhombus shaped dipole IR-nanoantenna on a dielectric substrate with $\mathrm{h}=150$ $\mathrm{nm}, \mathrm{w}=5 \mu \mathrm{m}, \mathrm{L}=9 \mu \mathrm{m}, \mathrm{L}_{\mathrm{s}}=5 \mu \mathrm{m}, \mathrm{t}=50 \mathrm{~nm}$ and gap $=100 \mathrm{~nm}$.

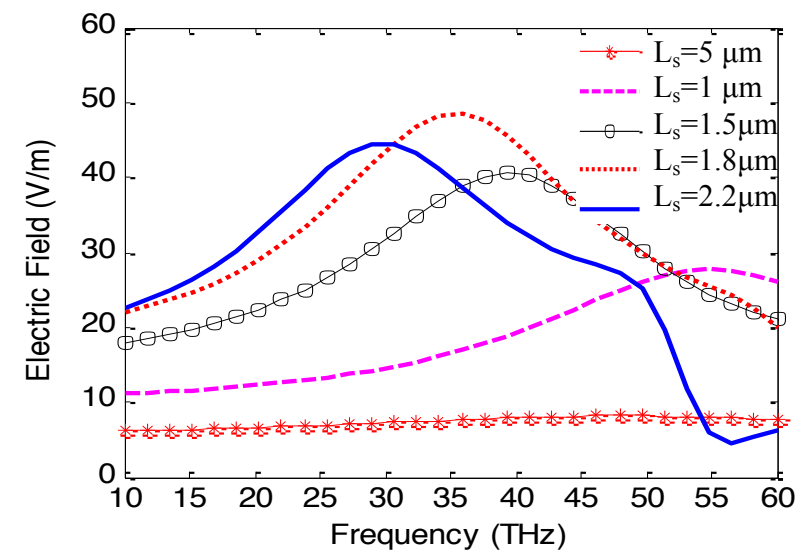

a.

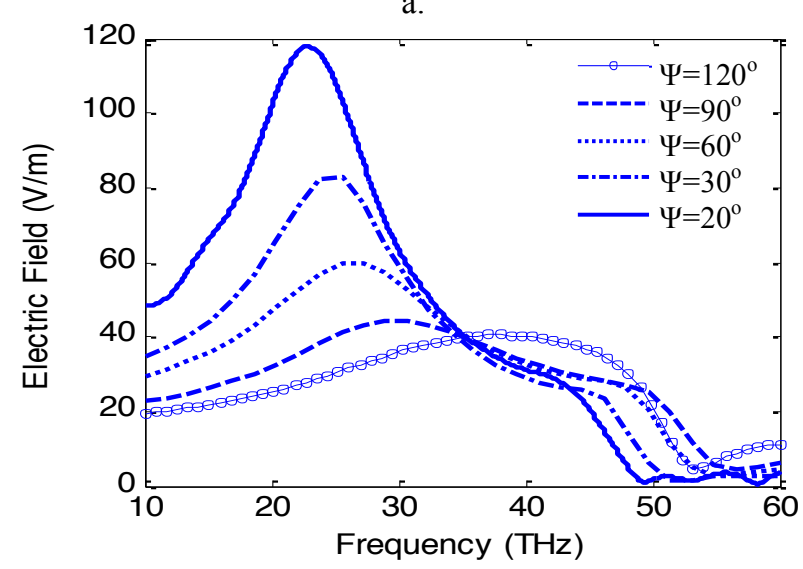

b.

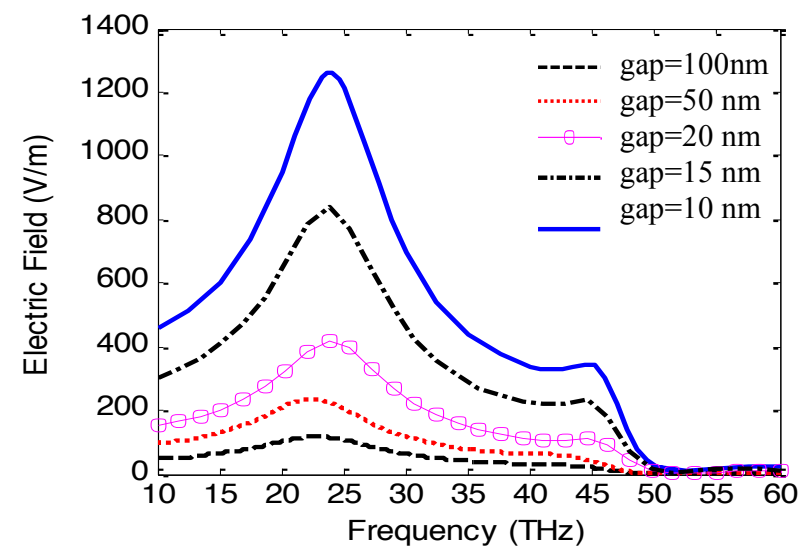

c.

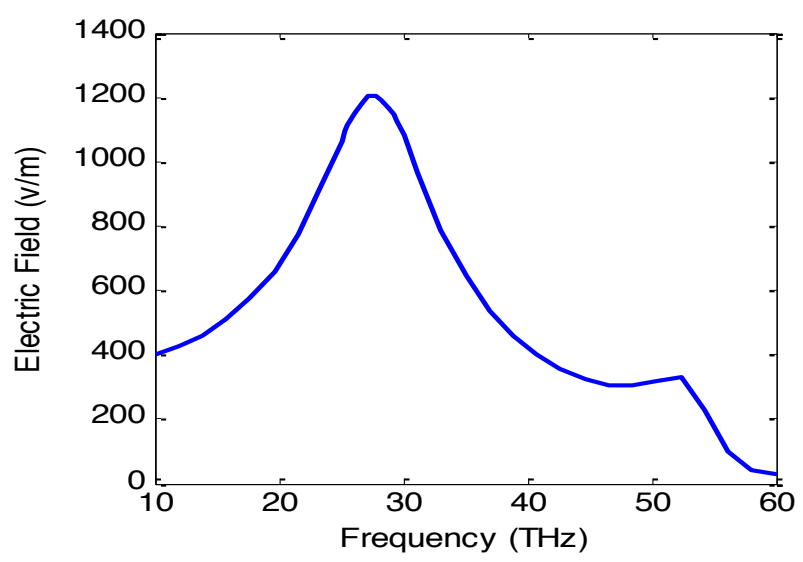

d.

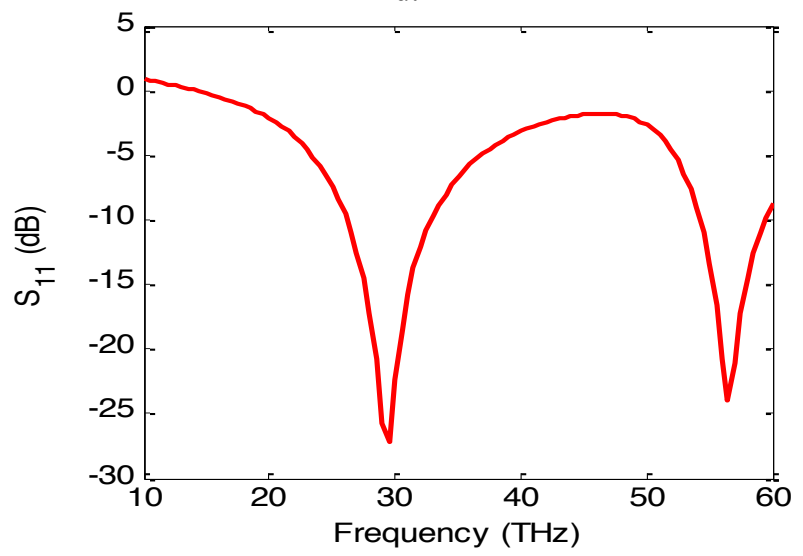

e.

Figure 3. The electric field variation versus frequency for rhombic dipole IR-nanoantenna on a dielectric substrate with $\mathrm{h}=150 \mathrm{~nm}, \mathrm{w}=5 \mu \mathrm{m}, \mathrm{L}=9 \mu \mathrm{m}, \mathrm{t}=50 \mathrm{~nm}$. a. $\psi=90^{\circ}$, gap $=100 \mathrm{~nm}$, and different values of $\mathrm{L}_{\mathrm{s}}, \mathrm{b} . \mathrm{Ls}=2.2 \mu \mathrm{m}$, gap $=100 \mathrm{~nm}$ and different values of tip angle $\psi$, c. Ls $=2.2$ $\mu \mathrm{m}, \Psi=20^{\circ}$ and different values of the gap, d. $\psi=20^{\circ}$, gap $=10 \mathrm{~nm}$, and $\mathrm{Ls}=1.8 \mu \mathrm{m}$, e. The magnitude of the Reflection coefficient for the case $\mathrm{d}$.

Figure 3.a shows the variation of near electric field intensity versus frequency for different arm lengths at $\Psi=90$ degrees and gap=100 $\mathrm{nm}$. An optimum rhombus side-length $\mathrm{L}_{\mathrm{s}}=2.2 \mu \mathrm{m}$ is achieved for maximum near field of $44.4 \mathrm{~V} / \mathrm{m}$ at $28.3 \mathrm{THz}$. In order to enhance the received electric field, the rhombus dipole tip angle is optimized to maximize the electric field across the gap. The variations of the electric field with frequency for different rhombus tip angles with $\mathrm{L}_{\mathrm{s}}=2.2 \mu \mathrm{m}$, and gap $=100 \mu \mathrm{nm}$ are shown in Fig.3b. Decreasing the tip angle $\Psi$ leads to increasing the charge accumulation across the gap which increases the electric field to $117.7 \mathrm{~V} / \mathrm{m}$ for $\Psi=20$ degrees at $\mathrm{L}_{\mathrm{s}}=2.2 \mu \mathrm{m}$, and gap $=100 \mathrm{~nm}$. To study the effect of the gap size on the value of the electric field across the gap, several gaps of $10 \mathrm{~nm}, 15$ $\mathrm{nm}, 20 \mathrm{~nm}, 50 \mathrm{~nm}$ and $100 \mathrm{~nm}$ have been used with a frequency sweep from 10 to $60 \mathrm{THz}$. Simulation results show that the electric field across the gap is increased with decreasing the gap due to the increase in charge accumulation, but with a very slight variation in the resonance frequency. The variations of the electric field with 
frequency for different gaps at $\Psi=20$ degrees and $L_{s}=2.2 \mu \mathrm{m}$ are shown in Fig.3c. The dipole shape of the IR nanoantenna has a large effect on the value of maximum received field. The maximum near field is achieved with $\Psi=20$ degrees, $\mathrm{L}_{\mathrm{s}}=2.2 \mu \mathrm{m}$ and a gap of $10 \mathrm{~nm}$. However, shorter gaps and narrower tip angle will produce higher field strength at the gap, but it will be quite difficult to handle. The rhombus IR nanoantenna has been optimized to move the peak point of the electric field from $23 \mathrm{THz}$ to $28.3 \mathrm{THz}$. The electric field and the reflection coefficient variation versus frequency for optimized rhombic dipole IRnanoantenna on a dielectric substrate with $\mathrm{h}=150 \mathrm{~nm}, \mathrm{w}=5$ $\mu \mathrm{m}, \mathrm{L}=9 \mu \mathrm{m}, \mathrm{t}=50 \mathrm{~nm}$, gap $=10 \mathrm{~nm}, \psi=20^{\circ}, \mathrm{L}_{\mathrm{s}}=1.8 \mu \mathrm{m}$ are shown in Fig. 3d, e. The electric field variations of the rhombus IR nanoannna using copper, silver and gold are shown in Fig. 4. The rhombus IR nanoantenna made from copper gives the highest electric field intensity, while the rhombus IR nanoantenna made from gold gives the lowest electric field intensity at $25 \mathrm{THz}$. The gold is selected throughout the paper because of the stability of conductivity characteristics. The second proposed dipole shape is cylindrical gold disk, printed on a Sio2 dielectric substrate designed to operate at $10.6 \mu \mathrm{m}$. Figure 5 shows the detailed construction of cylindrical shaped dipole IR nanoantenna on a dielectric substrate. The dimensions of the cylindrical dipole are radius $\mathrm{r}=1.5 \mu \mathrm{m}$, thickness $\mathrm{t}=50 \mathrm{~nm}$, gap of 100 $\mathrm{nm}$ and printed on Sio2 dielectric substrate of $9 \times 5 \times 0.15$ $\mu^{3}$. These dimensions are optimized to get maximum electric field at $28.3 \mathrm{THz}$.

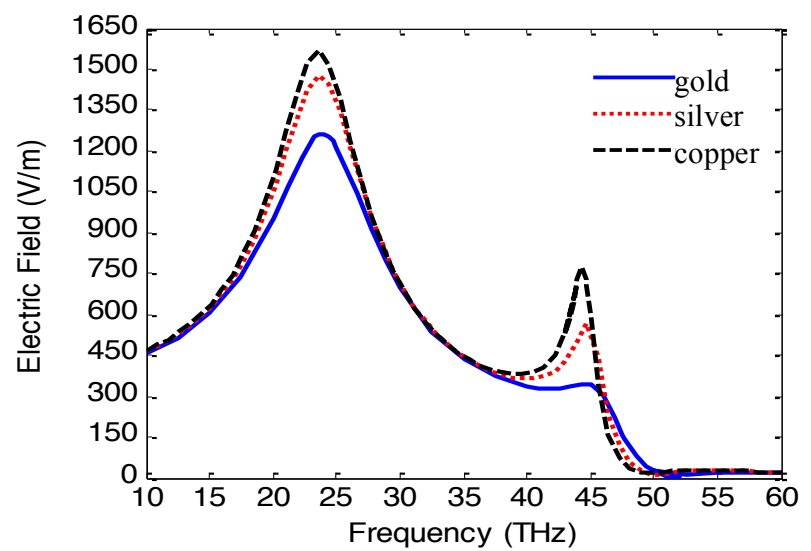

Figure 4. The electric field variation versus frequency for rhombic dipole IR-nanoantenna made from different metals printed on a dielectric substrate with $\mathrm{h}=150 \mathrm{~nm}, \mathrm{w}=5 \mu \mathrm{m}$, $\mathrm{L}=9 \mu \mathrm{m}, \mathrm{t}=50 \mathrm{~nm}, \mathrm{~L}_{\mathrm{s}}=2.2 \mu \mathrm{m}$, gap $=100 \mathrm{~nm}$ and $\psi=20^{\circ}$.

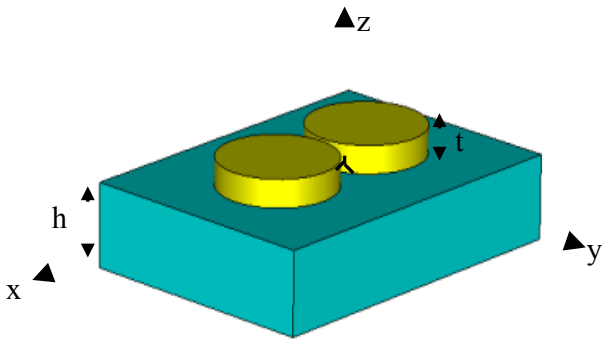

a. 3-D view

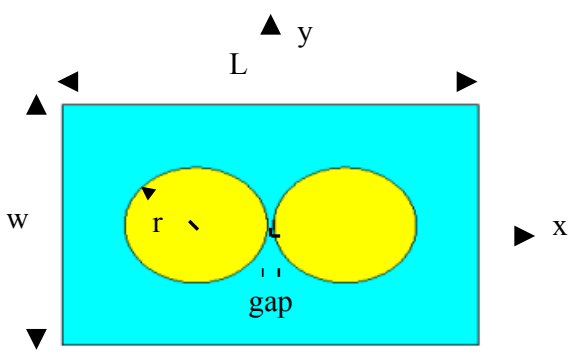

b. Top view

Figure 5. The detailed construction of cylindrical dipole IR nano-antenna with $\mathrm{h}=150 \mathrm{~nm}, \mathrm{w}=5 \mu \mathrm{m}, \mathrm{L}=9 \mu \mathrm{m}, \mathrm{r}=1.5 \mu \mathrm{m}$, $\mathrm{t}=50 \mathrm{~nm}$ and gap $=100 \mathrm{~nm}$.

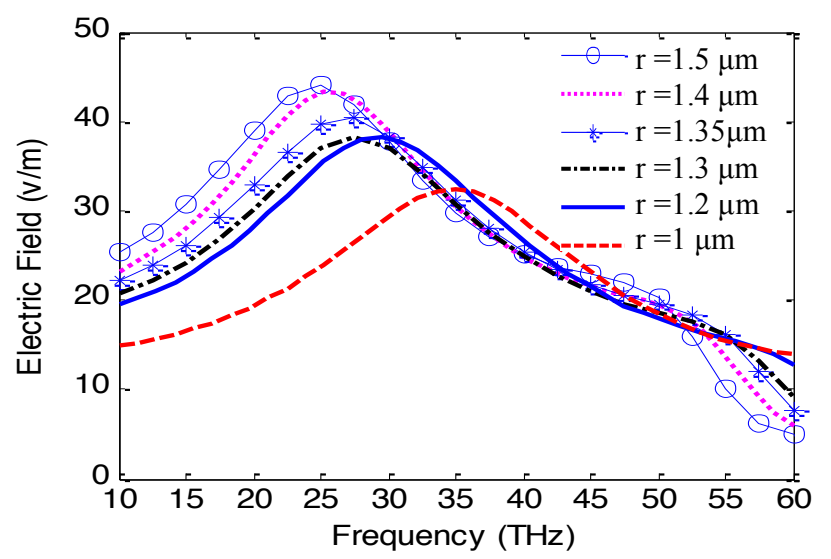

a.

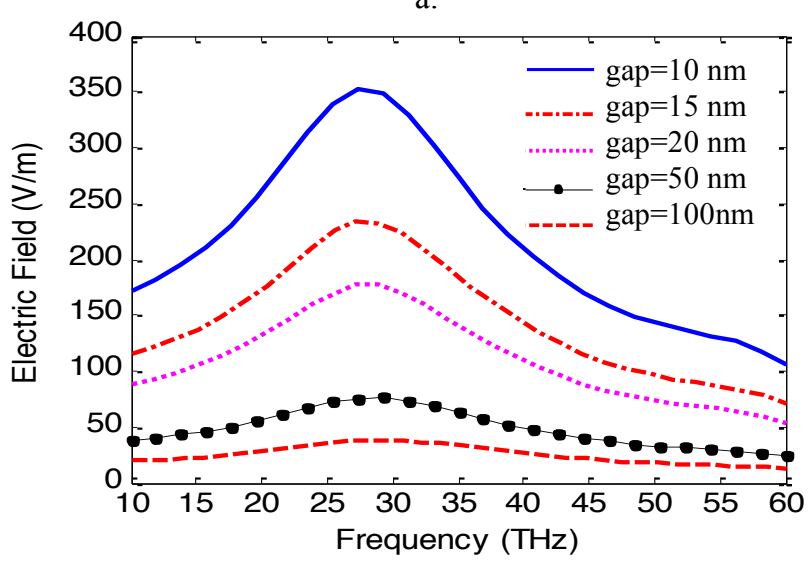

b.

Figure 6 a. The electric field variation versus frequency for cylindrical dipole with different values of cylindrical radius, b. The electric field variation versus frequency for cylindrical dipole with $\mathrm{h}=150 \mathrm{~nm}, \mathrm{w}=5 \mu \mathrm{m}, \mathrm{L}=9 \mu \mathrm{m}, \mathrm{t}=50$ $\mathrm{nm}, \mathrm{r}=1.2 \mu \mathrm{m}$ for different values of gap.

Figure 6 shows a parametric study for the nanoantenna dimensions to get maximum electric field at the dipole gap at $28.3 \mathrm{THz}$. A maximum electric field of $352.1 \mathrm{~V} / \mathrm{m}$ is achieved for $\mathrm{r}=1.2 \mu \mathrm{m}$ and a gap of $10 \mathrm{~nm}$. The induced voltage across the gap for rectangular, circular and rhombus shaped dipole arm nanoantennas are shown in Fig. 7, where $\mathrm{L}_{\text {rod }}=5 \mu \mathrm{m}$ and $\mathrm{W}_{\text {rod }}=0.5 \mu \mathrm{m}$ for rectangular dipole, radius $=1.2 \mu \mathrm{m}$ for wire of cylindrical dipole, and $\Psi=20$ degrees, $\mathrm{L}_{\mathrm{s}}=2.2 \mu \mathrm{m}$ for a rhombus arms dipole. The rhombus 
shaped IR nanoantenna dipole introduces maximum voltage of $12.5 \mu \mathrm{V}$ compared to $3.5 \mu \mathrm{V}$ for circular and $4.3 \mu \mathrm{V}$ for rectangular IR nanoantenna. The different dipole shapes are designed to run at $28.3 \mathrm{THz}$. The dimensions for each IR dipole structure are optimized for the highest electric field at 28.3 THz, the three shapes have thickness $\mathrm{t}=50 \mathrm{~nm}$ and printed on $\mathrm{SiO}_{2}$ dielectric substrate with dimensions of $9 \times 5 \times 0.15 \mu^{3}$. The solutions are calculated using the FIT and compared with that calculated using the FEM technique. Good agreement has been obtained. The electric and magnetic field distributions for different dipole shapes are shown in Fig. 8. The electric field is concentrated across the dipole gap for each case. The 3-D radiation pattern of rhombus IR nanoantenna is shown in Fig. 9. Omnidirectional radiation pattern is obtained in $\mathrm{x}-\mathrm{z}$ plane, while figure of eight patterns are obtained in the other principal planes.

\section{The effect of incident plane wave polarization}

The angle of incidence and the polarization of the incident electromagnetic field directly affect the voltage induced on the nanoantenna device. For normal incident plane wave propagating at angle $\theta=0$ and $\phi$ varies from 0 to 90 degrees, corresponding to electric field rotating from $\mathrm{x}-$ axis to y-axis is shown in Fig. 10a. The electric field intensity variation with frequency for rhombic IRnanoantenna with different electric field polarization angles are presented in Fig. 10b. The x-polarized plane wave $(\phi=0$ and $\theta=0$ ) introduces a maximum near field across the dipole gap with maximum field of $923.6 \mathrm{~V} / \mathrm{m}$ at $28.3 \mathrm{THz}$, while the y-polarized plane wave $(\phi=90$ and $\theta=0)$ gives the minimum received field. The electric filed intensity variations with plane wave electric field angle $\phi$ at $28.3 \mathrm{THz}$ for different IR-nanoantenna shapes are shown in Fig. 10c $\left(\mathrm{L}_{\mathrm{rod}}=5 \mu \mathrm{m}\right.$ for rectangular dipole, circular dipole with radius $=1.2 \mu \mathrm{m}$ and for rhombus has $\Psi=20$ degrees, $L_{s}=2.2 \mu \mathrm{m}$ ). The three shapes have thickness $\mathrm{t}=50 \mathrm{~nm}$ and printed on $\mathrm{Sio}_{2}$ dielectric substrate with dimensions of $9 \times 5 \times 0.15 \mu \mathrm{m}^{3}$.

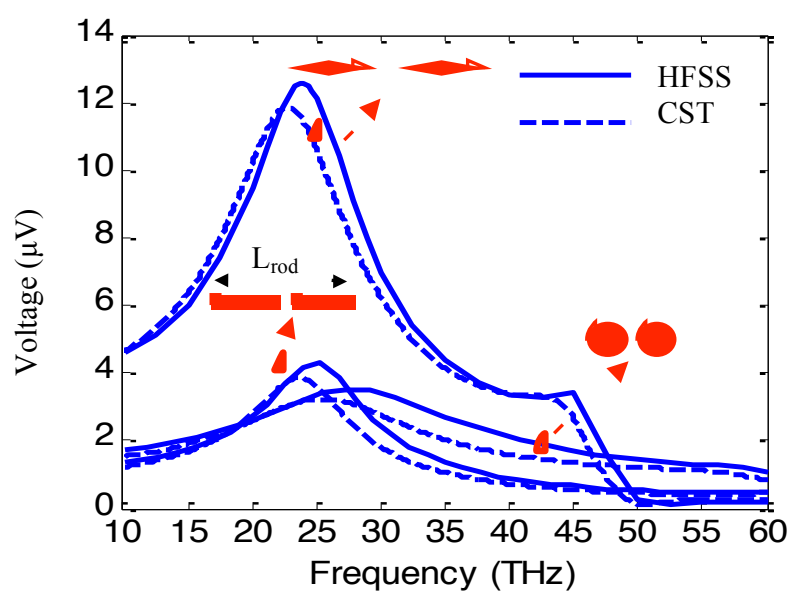

Figure 7. The voltage variation verses frequency for different dipole shapes.

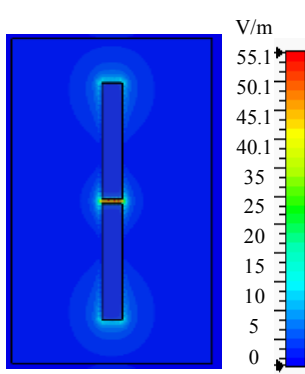

Electric field intensity

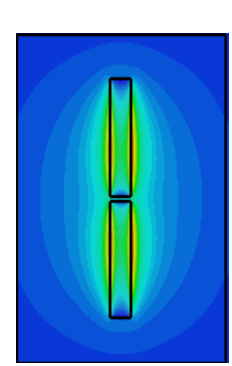

$\mathrm{A} / \mathrm{m}$

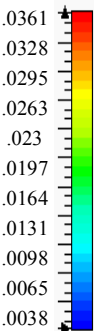

Magnetic field intensity

a. Rectangular

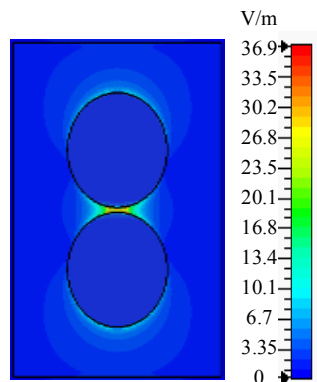

Electric field intensity

$$
\text { b. cylindrical }
$$

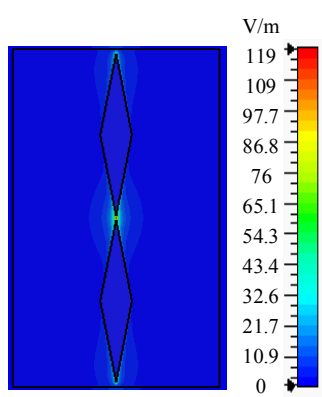

Electric field intensity

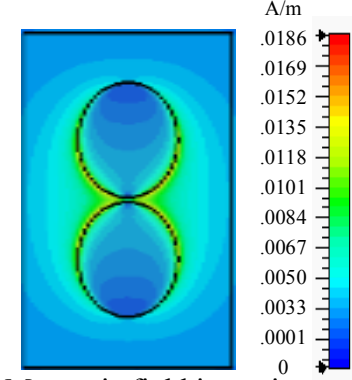

Magnetic field intensity

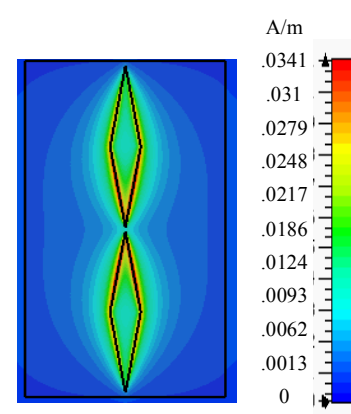

Magnetic field intensity

b. rhombic
Figure 8. The electric and magnetic field intensity distribution on different shapes of IR nanoantennas.

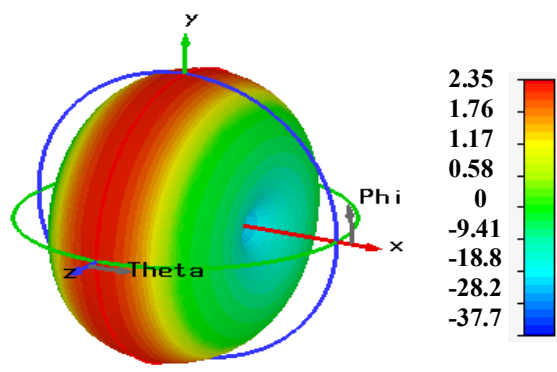

Figure 9. The 3-D radiation pattern of the rhombic dipole IR-nanoantenna on a dielectric substrate with $\mathrm{h}=150 \mathrm{~nm}$, $\mathrm{w}=5 \mu \mathrm{m}, \mathrm{t}=50 \mathrm{~nm}$, gap $=100 \mathrm{~nm}, \mathrm{~L}_{\mathrm{s}}=2.2 \mu \mathrm{m}$ at $28.3 \mathrm{THz}$.

The rhombic shape introduces a higher received electric field across the gap than the round and rectangular antennas. The electric field intensity is decreased with increasing the rotation angle of incident plane wave electric field. For oblique incident plane wave propagated at angle $\phi=0$ and $\theta$ 
varies from 0 to 90 degrees, corresponding to electric field rotating from $\mathrm{x}$-axis to $-\mathrm{z}$-axis as shown in Fig.11a. The electric field intensity variations with frequency for rhombic IR-nanoantenna at different electric field polarization are shown in Fig.11b. The maximum field intensity is decreased with increasing the incidence angle to reach its minimum value at $\theta=90$ degrees.

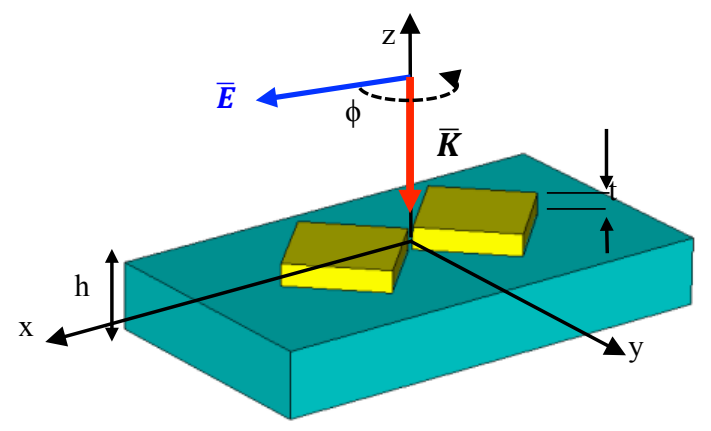

a.

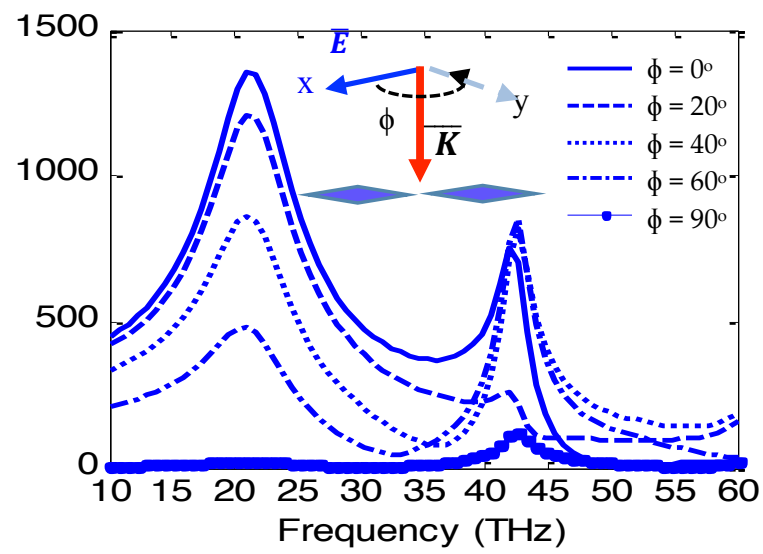

b.

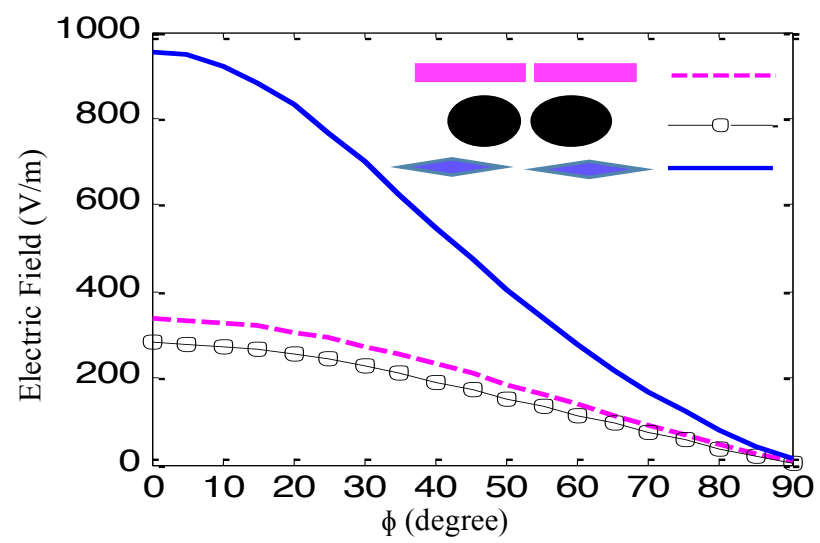

c.

Figure 10a. The angle of incidence and the polarization of the incident electromagnetic field directly affect the voltage induced on the nanoantenna device, $b$. The electric field variation versus $\phi$ for different shapes of the dipole IRnanoantenna on a dielectric substrate at $28.3 \mathrm{THz}, \mathrm{c}$. The electric field variation versus frequency for rhombic dipole IR-nanoantenna on a dielectric substrate with $\mathrm{h}=150 \mathrm{~nm}$, $\mathrm{w}=5 \mu \mathrm{m}, \mathrm{L}=5 \mu \mathrm{m}, \mathrm{t}=50 \mathrm{~nm}, \mathrm{gap}=10 \mathrm{~nm}, \mathrm{~L}_{\mathrm{s}}=2.2 \mu \mathrm{m}, \Theta=0$ and different values $\phi$.

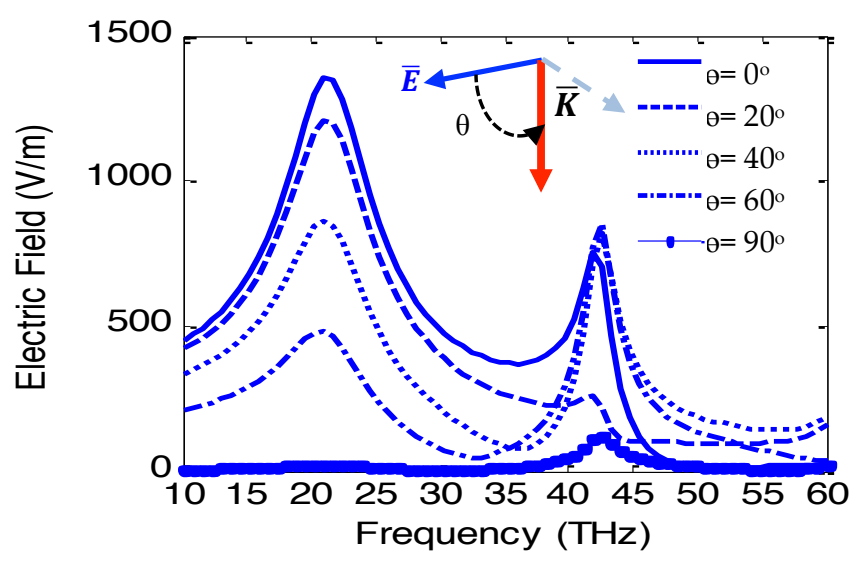

a.

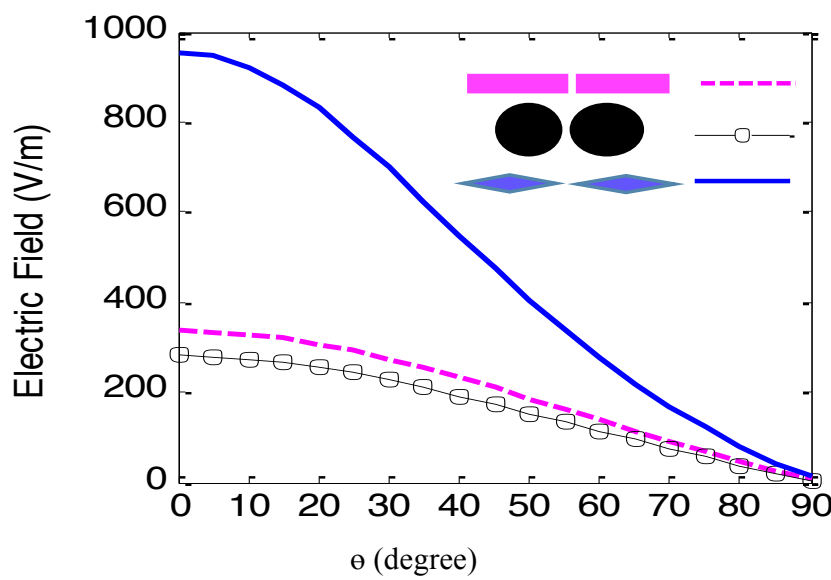

b.

Figure 11a. The electric field variation versus frequency for rhombic dipole IR-nanoantenna on a dielectric substrate with $\mathrm{h}=150 \mathrm{~nm}, \mathrm{w}=5 \mu \mathrm{m}, \mathrm{t}=50 \mathrm{~nm}, \mathrm{gap}=10 \mathrm{~nm}, \mathrm{~L}_{\mathrm{s}}=2.2 \mu \mathrm{m}$, $\phi=0$ and different values for $\theta, b$. The electric field variation versus $\Theta$ for different shapes of the dipole IR-nanoantenna on a dielectric substrate at $28.3 \mathrm{THz}$.

\section{Dual-Polarized Infrared Rectenna}

Dual polarized IR-nanoantenna has been designed which consist of 4 rhombic arms forming two crossed dipoles acting as an IR-nanoantenna on a dielectric substrate with $\mathrm{h}=150 \mathrm{~nm}, \mathrm{w}=9 \mu \mathrm{m}, \mathrm{L}=9 \mu \mathrm{m}, \mathrm{L}_{\mathrm{s}}=2.2 \mu \mathrm{m}, \mathrm{t}=50 \mathrm{~nm}$ and gap $=10 \mathrm{~nm}$ as shown in Fig.12. Figure 13a, Figure 13b shows the electric field variations versus frequency for 4 rhombic arms crossed dipole IR-nanoantenna on a dielectric substrate getting the same value of the electric field at the gap using different polarization $\left(\mathrm{E}_{\mathrm{y}}, \mathrm{E}_{\mathrm{x}}\right)$. In both figures the electric field variation for normal incident plane wave with electric field polarized in $\mathrm{x}$-direction and in the $\mathrm{y}$-direction respectively. The two arms are identical to cross polarized field response. Arm $(1,3)$ is co-polarized with $\overline{\boldsymbol{E}}$ in $\mathrm{X}$-axis and cross-polarized with the y-axis. Arm $(2,4)$ is copolarized with $\overline{\boldsymbol{E}}$ in y-axis and cross-polarized with the xaxis. 


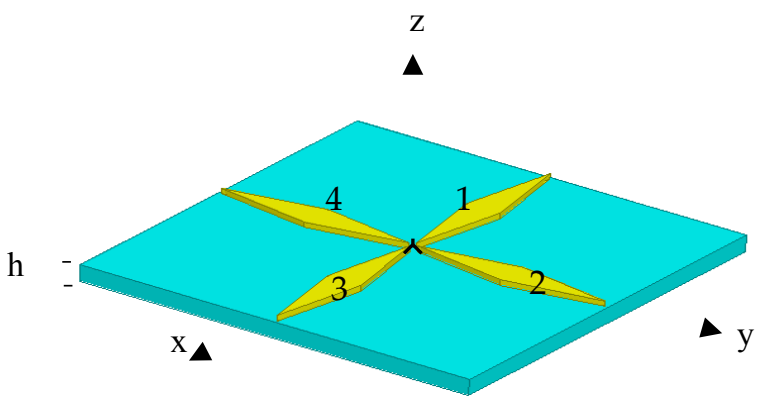

a. 3-D view

$\Delta \mathrm{y}$

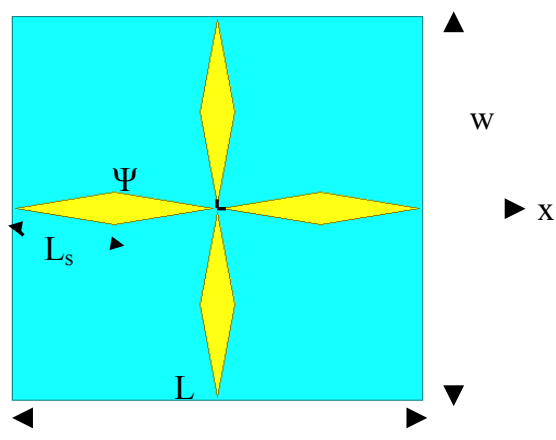

b. Side view

Figure 12. The detailed construction of the rhombic dipole IR-nanoantenna on a dielectric substrate with $\mathrm{h}=150 \mathrm{~nm}$, $\mathrm{w}=9 \mu \mathrm{m}, \mathrm{L}=9 \mu \mathrm{m}, \mathrm{L}_{\mathrm{s}}=2.2 \mu \mathrm{m}, \mathrm{t}=50 \mathrm{~nm}$ and gap $=10 \mathrm{~nm}$.

\section{The IR-Nanoantenna dipole with MIM diode}

The MIM diode is used for IR rectification with the IRnanoantenna. The diode incorporates an insulating layer between two electrodes thin enough to allow tunnel conduction $[2,14]$. The performance of the MIM diode is based on three main characteristics. The first is the diode's responsivity which is a measure of diode's rectification ability. The second is the diode's resistance which affects the matching between the antenna and the rectifier. The third is the diode's cut-off frequency and is given by

$$
f_{c}=\frac{1}{2 \pi R_{D} C_{D}} \quad \text { where } C_{D}=\varepsilon_{O} \varepsilon_{r} \frac{A}{d}
$$

where $R_{D}$ is the equivalent resistance of the diode, $C_{D}$ is the MIM's capacitance, $\varepsilon_{\mathrm{r}}$ is the relative dielectric constant, and $\mathrm{d}$ is the thickness of the dielectric. A is the overlap or contact area. By increasing the dielectric thickness, the capacitance decreases and the cut-off frequency increases. However, the diode's resistance is increased, since the number of tunnelling electrons decreases exponentially as the diode's thickness increases [15]. The resistance of the rectifiers was around $300 \mathrm{~K} \Omega$ for with small junction areas [9]. These characteristics give a good response advantage in the performance of this kind of junctions at the infrared applications [14]. The $300 \mathrm{k} \Omega$ devices were chosen since they offered the best resistance/sensitivity trade-off for MIM diodes.

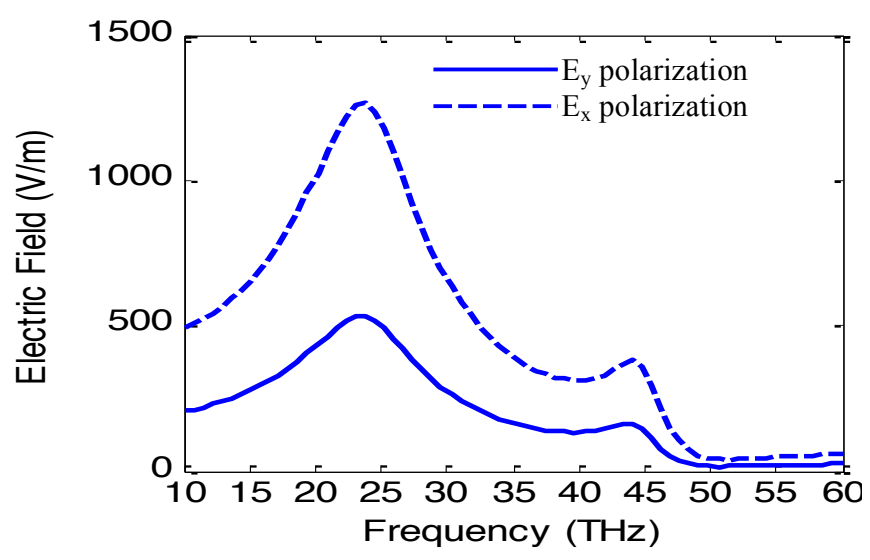

a.

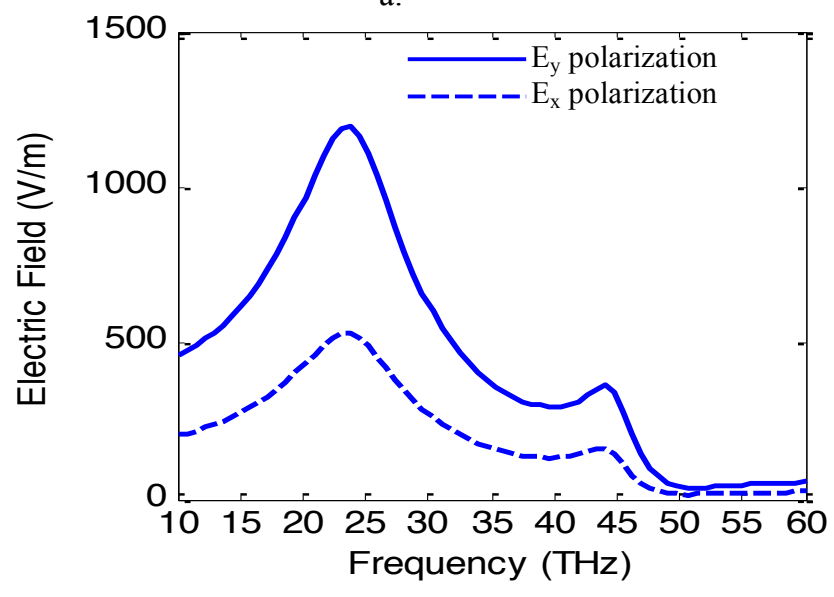

b.

Figure 13a. The electric field variation versus frequency for rhombic dipole IR-nanoantenna on a dielectric substrate with $\mathrm{h}=150 \mathrm{~nm}, \mathrm{w}=9 \mu \mathrm{m}, \mathrm{L}=9 \mu \mathrm{m}, \mathrm{t}=50 \mathrm{~nm}$, gap=10 nm, $\mathrm{L}_{\mathrm{s}}$ $=2.2 \mu \mathrm{m}$ for $(1,3), \mathrm{b}$. The electric field variation versus frequency for rhombic dipole IR-nanoantenna on a dielectric substrate with $\mathrm{h}=150 \mathrm{~nm}, \mathrm{w}=5 \mu \mathrm{m}, \mathrm{t}=50 \mathrm{~nm}$, gap $=10 \mathrm{~nm}, \mathrm{~L}_{\mathrm{s}}$ $=2.2 \mu \mathrm{m}$ for $(2,4)$.

In order to integrate the MIM diode with the antenna, one end of the rhombic dipole's arm has been overlapped, to provide a room for the diode. The two arms of the rhombic dipole are overlapped over an area of $60 \times 60 \mathrm{~nm}^{2}$, with an insulator layer with dielectric constant of $\varepsilon_{\mathrm{r}}=8$ and $\mathrm{a}$ thickness of $8.5 \mathrm{~nm}$ has been inserted within the gap region as shown in Fig. 14a. The variation of the input impedance versus frequency for different overlapping areas are shown in Fig. 14b. The real and imaginary part of the input impedance is decreased by increasing the overlapping area with a slight shift down in maximum frequency. The variations of the electric field intensity over the IR spectrum for rhombic shape IR-nanoantenna at different MIM overlapping area are shown in Fig. 15a. By decreasing the overlapping areas, the diode capacitance is decreased and the electric field intensity is enhanced to maximum value of $1475.6 \mathrm{~V} / \mathrm{m}$ for MIM with area $20 \times 20 \mathrm{~nm}^{2}$. The variations of the radiation efficiency versus frequency for different overlapping areas are shown in Fig. 15b. The radiation efficiency is decreased with decreasing the overlapping area and varies around $80 \%$ to $92 \%$. 


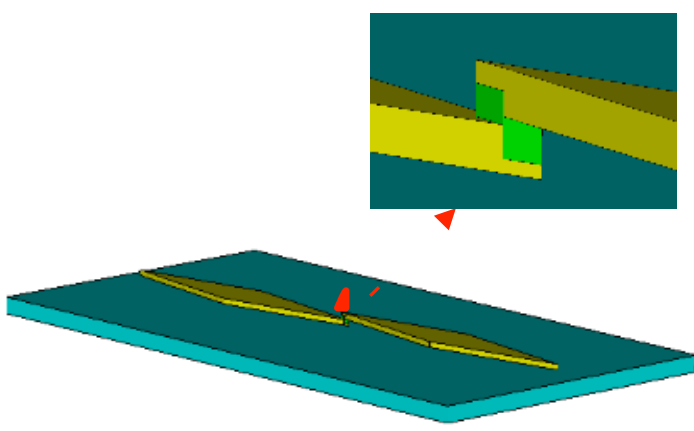

a.

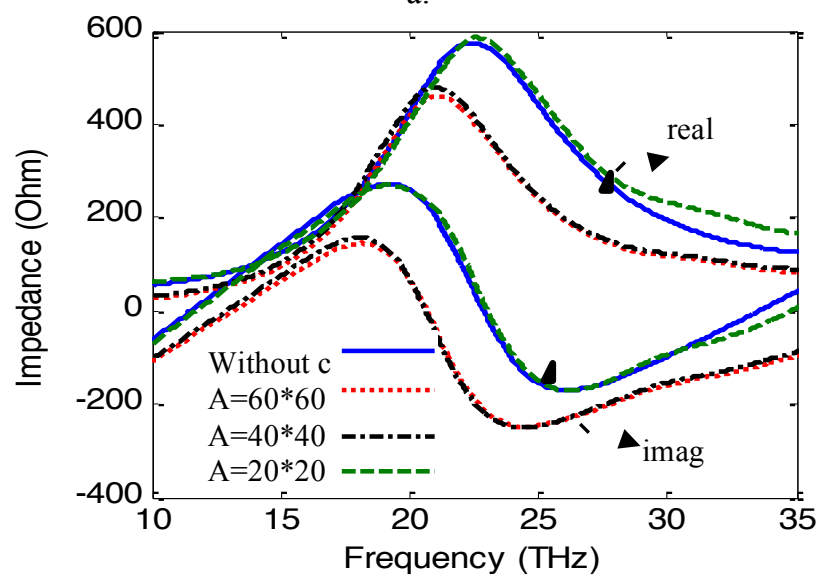

b.

Figure 14. A rhombic dipole IR-nanoantenna on a dielectric substrate overlapped area to construct MIM diode, b. The impedance variation versus frequency for rhombic dipole IR-nanoantenna on a dielectric substrate without overlapping area and with different overlapping area using insulator of $\varepsilon_{r}=8$.

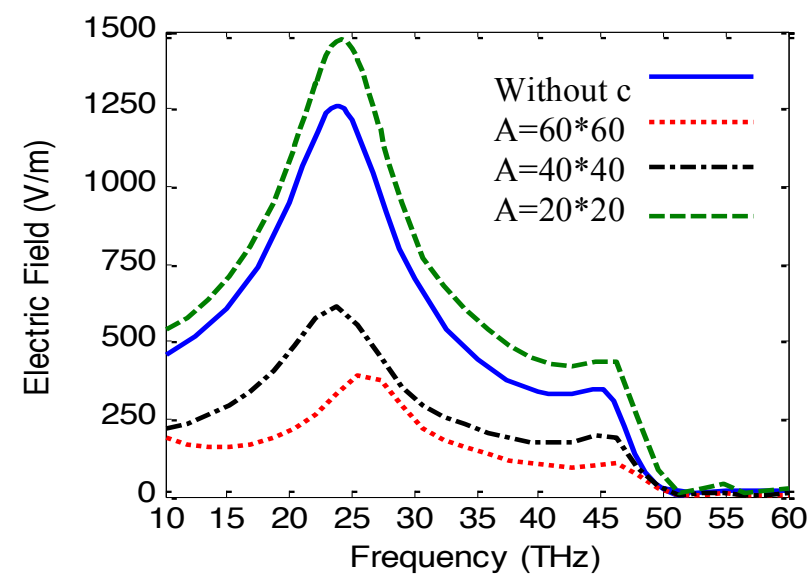

a.

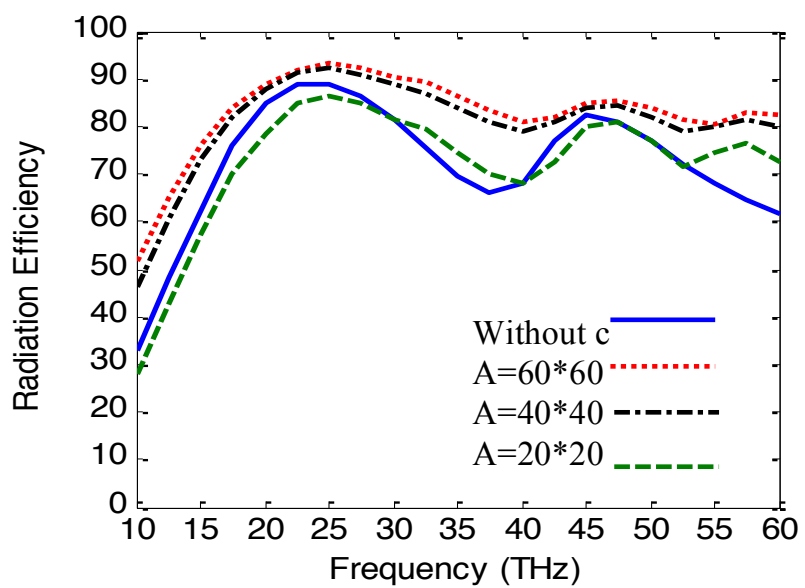

b.

Figure 15a. The electric field variation versus frequency for rhombic dipole IR-nanoantenna with different overlapping area using insulator of $\varepsilon_{r}=8, \mathrm{~b}$. The radiation efficiency variation versus frequency for rhombic dipole IRnanoantenna.

\section{Conclusions}

A linearly-polarized receiving mode nano-antenna is designed for solar energy harvesting. A rhombic nanoantenna dipole of gold printed on a silicon dioxide substrate is investigated. The dimensions of the dipole are optimized for maximum near electric field intensity at a frequency around $28.3 \mathrm{THz}$. The shape of the dipole IR nanoantenna has a great effect on the value of maximum received field. The maximum near field intensity of 1260 $\mathrm{v} / \mathrm{m}$ is achieved with $\Psi=20^{\circ}, \mathrm{L}_{\mathrm{s}}=2.2 \mu \mathrm{m}$ and a gap of $10 \mathrm{~nm}$ without MIM diode. Dual polarized IR-nanoantenna has been designed which consists of 4 rhombic arms crosseddipole IR-nanoantenna on a dielectric substrate with $h=150$ $\mathrm{nm}, \mathrm{w}=9 \mu \mathrm{m}, \mathrm{L}=9 \mu \mathrm{m}, \mathrm{L}_{\mathrm{s}}=2.2 \mu \mathrm{m}, \mathrm{t}=50 \mathrm{~nm}$ and gap=10 nm getting the same value of the electric field at the gap using different polarization $\left(\mathrm{E}_{\mathrm{y}}, \mathrm{E}_{\mathrm{x}}\right)$. The Metal Insulator Metal (MIM) diode is incorporated with the dipole nano-antenna to rectify the received voltage. The radiation efficiency of the solar energy collector with integrated MIM diode has been introduced. The MIM diode resistance and capacitance have been calculated to be $\mathrm{R}_{\mathrm{D}}=3828 \Omega, \mathrm{C}_{\mathrm{D}}=376 \mathrm{nF}$.

\section{References}

[1] M. Galloa, L. Mescia, O. Losito, M. Bozzetti, and F. Prudenzano "Design of optical antenna for solar energy collection," Energy, vol.39, no.1, pp. 27-32, March 2012.

[2] I.E. Hashem, N.H. Rafat, and E.A. Soliman "Dipole nantennas terminated by traveling wave rectifiers for ambient thermal energy harvesting," IEEE Transactions on Nanotechnology, vol.13, no.4, pp.767 - 778, 2014.

[3] Hend A. Malhat, Nermeen A. Eltresy, Saber H. ZainudDeen, and Kamal H. Awadalla, "Nano-dielectric resonator antenna reflectarray/ transmittarray for 
terahertz applications," Advanced Electromagnetics, vol. 4, no. 1, pp. 36-44, June 2015.

[4] Hend A. Malhat, Nermeen A. Eltresy, Saber H. ZainudDeen, and Kamal H. Awadalla, "Reflectarray nanodielectric resonator antenna using different metals," The Applied Computational Electromagnetics Society Journal, vol. 30, no. 9, pp. 967-974, Sept. 2015.

[5] M. Klemm, "Novel directional nanoantennas for singleemitter sources and wireless nano-links," International Journal of Optics, vol.2012, pp. 1-7, 2012.

[6] S. Maksymov, A.E. Miroshnichenko, and Y.S. Kivshar, "Actively tunable bistable optical Yagi-Uda nanoantenna," Opt. Express, vol. 20, no. 8, pp. 89298938, 2012.

[7] M. Agio, "Optical antennas as nanoscale resonators," Nanoscale, vol.4, pp. 692-706, 2012.

[8] S.A. Maier, Plasmonics fundamentals and applications, Springer, USA, 2007.

[9] A.M. A. Sabaawi, C.C. Tsimenidis, and B.S. Sharif, "Analysis and modeling of infrared solar rectennas," IEEE Journal of Selected Topics in Quantum Electronics, vol. 19, no. 3, DOI: 10.1109/JSTQE.2012.2227686, 2013.

[10]I. Kocakarin, K. Yegin "Glass superstrate nanoantennas for infrared energy harvesting applications" International Journal of Antennas and Propagation, vol. 2013, pp. 1-7, 2013.

[11]D.K. Kotter, S.D. Novack, W.D. Slafer, and P.J. Pinhero, "Theory and manufacturing processes of solar nanoantenna electromagnetic collectors," Journal of Solar Energy Engineering, vol. 132, no. 1, pp. 1-8, Jan. 2010.

[12]S. J. Cooke, R. Shtokhamer, A.A. Mondelli, and B. Levush, "A fnite integration method for Conformal, structure-grid, electromagnetic simulation," Journal of Computational Physics, vol. 215, no. 1, pp. 321-347, 2006.

[13]N. Kumar, Spontaneous Emission Rate Enhancement Using Optical Antennas; Ph.D. Thesis, University of California, Berkeley, USA, 2013.

[14]I.E. Hashem, Infrared Solar Energy Harvesting Using Nano-Rectennes. M.Sc. Faculty of Engineering, Cairo University, Giza, Egypt, 2013.

[15]M.N. Gadalla, M. Abdel-Rahman and A. Shamim, "Design, optimization and fabrication of a $28.3 \mathrm{THz}$ nano-rectenna for infrared detection and Rectification," Nature, Scientific Reports, vol. 4, pp. 1-9, Mar. 2014. 